\title{
STOCK MARKET DYNAMICS CREATED BY INTERACTING AGENTS
}

\author{
MOHAMED RIAD REMITA AND KARL-THEODOR EISELE
}

Received 19 March 2004; Revised 26 September 2005; Accepted 26 September 2005

We study a stock market model, consisting in a large number of agents, going eventually to infinity, and evaluate the stock price under the influence of opinions of different agents. Next we study the behavior of prices when the market is very nervous; there appear discontinuities (phase transitions) which can be interpreted as stock market crashes.

Copyright (c) 2006 M. R. Remita and K.-T. Eisele. This is an open access article distributed under the Creative Commons Attribution License, which permits unrestricted use, distribution, and reproduction in any medium, provided the original work is properly cited.

\section{Introduction}

Since the work of Samuelson [4], in which he used the geometric Brownian motion to explain the evolution of stock prices, and starting from the model of Black and Scholes [1], which was based on the preceding result to give the formula of evaluation of options, many were interested to develop new models without losing sight of the original work. We will refer to these two results to develop this work.

In an open market, a price is the result of a balance between offer and demand, that is, a stable compromise between the wishes of several people, called agents. The dynamic evolution of a price, as a stock exchange curve represents it, shows phenomena which are explicable only by incorporating the formation of such a balance. The agents react in a way rather independent with respect to received information of outside, but interact between them in the decision-making (the interaction is here of mean-type field, because the agents do not form a geometrical structure in a natural way). Such a market is inevitably incomplete.

A first question arises naturally: what will occur if the number of agents becomes very large? In the direction of the law of the large numbers, we can see that we have a convergence of the incomplete markets towards a complete market, of which the dynamic curve of price is independent of the individual decision of each agent, but which always reflects the global structures of the decision-making. 
A second aspect is essential. The individual perception of the market by an agent and its corresponding reaction is a microeconomic quantity. On the other hand, the stock exchange curves and especially the curves of the indices are macroeconomic quantities. Thus, at the same moment, we are interested to study in this model the transition from the microeconomic structures to macroeconomic quantities.

A number of questions may be asked; for example, which phenomena are explained by the transition from the microeconomic structures and which are purely macroeconomic? In particular, which part of volatility is the result of the microeconomic fluctuations and which part is purely macroeconomic volatility? Or also, generally the microeconomic or macroeconomic reactions have very different scales speed. Which is the influence of the quotient of these two speeds on the market?

A third aspect has a more mathematical interest: the external information, based on the economic situation, of the national or international events, is represented by a random process. This information influences the decisions of the agents. We are in the presence of a dynamic system evolving in a random environment. This has consequences for the fluctuations and the principles of great deviations.

For all these reasons, we will be brought to develop a model taking into account all the influences on the market in order to better interpret certain situations and try to answer some of the questions above.

We begin our paper in Section 2 by describing the model. Then in Section 3, we study the convergence of the empirical measure and the price process when $n$ goes to infinity. Finally, in Section 4, we study the evolution of the price process when the market is very nervous and give some examples.

\section{Construction of the model}

We suppose the model composed from agents, noted $i$ with $i=1, \ldots, n$; ( $n$ going eventually to infinity). Each agent has his personal opinion about the future evolution of the stock price on the market. The opinion is built individually from information about the enterprise whose stock is regarded. It depends also on external events, general circumstances, economic indicators, inflation, budget deficit, money supply, raw materials' prices, and behavior of other stock markets. This opinion is expressed by an individual drift depending on time, which will be noted by

$$
\mu_{i}(t)
$$

This means that the agent $i$ perceives the future evolution of the stock price given by

$$
S_{i, t}=S_{0} \mathscr{E}\left(X_{t}\right) \exp \left\{\mu_{i}(t) \cdot t\right\}
$$

with

$$
\mathscr{E}\left(X_{t}\right)=\exp \left\{X_{t}-\frac{1}{2}[X, X]_{t}^{c}\right\} \prod_{0<s \leq t}\left(1+\Delta X_{s}\right) \exp \left\{-\Delta X_{s}\right\}
$$


the stochastic exponential of the semimartingale $X_{t}$, which is solution of the following stochastic differential equation:

$$
\mathscr{E}\left(X_{t}\right)=1+\int_{0}^{t} \mathscr{E}\left(X_{s^{-}}\right) d X_{s}
$$

where $[X, X]_{t}^{c}$ is the continuous part of the bracket $[X, X]_{t}$ and we suppose that $\Delta X_{s}>-1$ for all $t \in[0, T] . S_{0}$ is the actual price of the stock, $\mu_{i}(t)$ the subjective opinion of the agent $i$ on the future evolution of the stock price, and $X_{t}$ the external stochastic perturbation. $X_{t}$ represents all external stock market data that have direct influence on prices, it will be for example economists' and politicians' opinions. We suppose $X_{0}=0$. Then the sequence of price processes $\left(S_{i, t}\right)_{i=1, \ldots, n}$ is a family of processes in a random environment.

At time $t$ the price $S_{t}^{n}$ of the stock is the result of the estimations of the $n$ agents; more precisely, it should be the median of the agents' estimations $S_{i, t}$ of agents $i$, weighted by the quantities of stocks offered or required by agent $i$. Agent $i$ buys stocks if the official price $S_{t}^{n}$ on the market is less than the individual price $S_{i, t}$ and he sells if $S_{t}^{n} \geq S_{i, t}$. Then the market is in equilibrium if $S_{t}^{n}$ is the weighted median of the $S_{i, t}$.

Moreover, we suppose that in the case $S_{t}^{n} \leq S_{i, t}$ the offered quantities are equal to $\psi\left(S_{i, t}\right)-\psi\left(S_{t}^{n}\right)$, and in the case $S_{t}^{n} \geq S_{i, t}$, the required quantities by the agent $i$ are equal to $\psi\left(S_{t}^{n}\right)-\psi\left(S_{i, t}\right)$, where $\psi$ is an increasing function in $\mathscr{C}^{2}\left(\mathbb{R}_{+}\right)$. Then the equilibrium of the market is given by

$$
\psi\left(S_{t}^{n}\right)=\frac{1}{n} \sum_{i=1}^{n} \psi\left(S_{i, t}\right) .
$$

In order to make the proofs easy, we will only study the case where $\psi(x)=x$, general case is easy to check.

We consider now how the subjective price of a stock is estimated by an agent $i$. Under the influence of the opinions of the other agents and external news, the agent will change his opinion from time to time; this is done by a change of his individual drift $\mu_{i}(t)$. The motivations of this change will be explained by interaction between the agents and also by the influence of the circumstances, this means that each agent will interpret the decision of the other agent like a new information, which can be known by publications, bank consultants, and in particular, by the stock price itself.

For our model, let us consider the vector

$$
\mu^{(n)}(t)=\left(\mu_{1}(t), \ldots, \mu_{n}(t)\right)
$$

which is a pure jump process and will represent the configuration of the opinions. We set $\mu^{(n)}(t) \in B^{n}$, where $B$ is a finite, closed interval of $\mathbb{R}$.

The external perturbation process $X_{t}$ is supposed to be an adapted process satisfying

$$
\begin{gathered}
\mathbb{E}\left[\sup _{t \leq T} \exp \left\{\lambda X_{t}\right\}\right] \leq C_{\lambda}<+\infty \quad \forall \lambda \in \mathbb{R}, \\
\Delta X_{t}>-1 \quad \forall t \in[0, T] .
\end{gathered}
$$


4 Stock market dynamics created by interacting agents

Conditionally to $X_{[0, T]}$, the pure jump process $\left(\mu^{(n)}(t)\right)_{0 \leq t \leq T}$ has the conditional infinitesimal generator $A=A\left(X_{t}\right)$ defined on product functions $f\left(b_{1}, \ldots, b_{n}\right)=\prod_{i=1}^{n} f_{i}\left(b_{i}\right)$, $f_{i} \in \mathscr{C}_{b}(B)$, by

$$
\begin{aligned}
&(A f)\left(\mu^{(n)}(t)\right)=\sum_{i=1}^{n}\left(\prod_{j \neq i} f_{j}\left(\mu_{j}(t)\right) \cdot \int_{B}\left[f_{i}(m)-f_{i}\left(\mu_{i}(t)\right)\right]\right. \\
&\left.\cdot \exp \left\{\alpha+\frac{1}{n} \sum_{k=1}^{n} g\left(m, \mu_{k}(t)\right)+m \cdot X_{t}\right\} \rho(d m)\right),
\end{aligned}
$$

with $\alpha$ a positive constant and $g \in \mathscr{C}_{b}\left(B^{2}\right)$.

The number $\alpha$ represents the level of the nervousness of the market, (general intensity of the agents to change their mind). The term $(1 / n) \sum_{k=1}^{n} g\left(m, \mu_{k}(t)\right)$ represents the interaction with the other agents. $m \cdot X_{t}$ is the influence of external events. The probability measure $\rho$ is the unbiased distribution of the new drift.

Conditionally to $X=X_{[0, T]}$, there exists a unique Markov process $\mathbb{P}^{n}(\cdot \mid X)$ on the Skorokhod space $\mathscr{D}\left(\mathbb{R}_{+}, B^{n}\right)$, the space of càdlàg processes with values in $B^{n}$.

Let $\mathbb{P}^{n}$ be the process defined on the space $\Omega=\mathscr{D}\left(\mathbb{R}_{+}, B^{n} \cdot \mathbb{R}\right)$. Then, we have for $f\left(b_{1}, \ldots, b_{n}\right)=\prod_{i=1}^{n} f_{i}\left(b_{i}\right), f_{i} \in \mathscr{C}_{b}(B)$,

$$
\begin{aligned}
& M_{t}^{n}= f\left(\mu^{(n)}(t)\right)-f\left(\mu^{(n)}(0)\right) \\
&-\int_{0}^{t} \sum_{i=1}^{n}\left(\prod_{j \neq i} f_{j}\left(\mu_{j}(s)\right) \cdot \int_{B}\left[f_{i}(m)-f_{i}\left(\mu_{i}(s)\right)\right]\right. \\
&\left.\cdot \exp \left\{\alpha+\frac{1}{n} \sum_{k=1}^{n} g\left(m, \mu_{k}(s)\right)+m \cdot X_{s}\right\} \rho(d m)\right) d s,
\end{aligned}
$$

a $\mathbb{P}^{n}$-martingale, conditionally to $X$.

Writing

$$
M_{t}^{n}=\int_{0}^{t} \int_{B^{n}}\left[f(\bar{m})-f\left(\mu^{(n)}\left(s^{-}\right)\right)\right] \tilde{\Lambda}(d \bar{m}, d s),
$$

where $\bar{m} \in B^{n}$,

$$
\begin{aligned}
& \tilde{\Lambda}(d \bar{m}, d s)=\Lambda(d \bar{m}, d s)-\sum_{i=1}^{n} \int_{B} \delta_{\left(\mu_{1}(s), \ldots, \mu_{i-1}(s), m, \mu_{i+1}(s), \ldots, \mu_{n}(s)\right)} \\
& \cdot \exp \left\{\alpha+\frac{1}{n} \sum_{k=1}^{n} g\left(m, \mu_{k}(s)\right)+m \cdot X_{s}\right\} \rho(d m) d s,
\end{aligned}
$$


$\Lambda(d \bar{m}, d s)$, conditionally to $X$, is a pure point process, the corresponding increasing process of $M_{t}^{n}$ will be given by

$$
\begin{aligned}
&\left\langle M^{n}, M^{n}\right\rangle_{t}=\int_{0}^{t} \int_{B^{n}}[\left.f(\bar{m})-f\left(\mu^{(n)}(s)\right)\right]^{2} \\
& \cdot\left(\sum_{i=1}^{n} \int_{B} \delta_{\left(\mu_{1}(s), \ldots, \mu_{i-1}(s), m, \mu_{i+1}(s), \ldots, \mu_{n}(s)\right)}\right. \\
&\left.\cdot \exp \left\{\alpha+\frac{1}{n} \sum_{k=1}^{n} g\left(m, \mu_{k}(s)\right)+m \cdot X_{s}\right\} \rho(d m)\right) d \bar{m} d s .
\end{aligned}
$$

\section{The study of the price process}

3.1. Convergence of the empirical measure. Define the empirical measure $\mu_{t}^{n}$ by

$$
\mu_{t}^{n}=\frac{1}{n} \sum_{i=1}^{n} \delta_{\mu_{i}(t)} \in \mathscr{P}(B),
$$

and endow $\mathscr{P}(B)$, the set of probability measures on the compact $B$, with the weak-star topology, for which it is compact. Now, for a continuous function $f \in \mathscr{C}(B)$, the process

$$
\left\langle f, \mu_{t}^{n}\right\rangle=\frac{1}{n} \sum_{i=1}^{n} f\left(\mu_{i}(t)\right)
$$

has the compensator (the previsible dual projection)

$$
\int_{B}\left[\left\langle f, \delta_{m}\right\rangle-\left\langle f, \mu_{t}^{n}\right\rangle\right] C\left(m, \mu_{t}^{n}, X_{t}\right) \rho(d m)
$$

where

$$
C^{n}\left(m, \mu_{t}^{n}, X_{t}\right)=\exp \left\{\alpha+\left\langle g(m, \cdot), \mu_{t}^{n}\right\rangle+m \cdot X_{t}\right\}
$$

This can be seen by applying the linear operator $A$ to the expression $(1 / n) \sum_{i=1}^{n} h^{l}(\bar{m})$ with $h^{l}(\bar{m})=f\left(m_{l}\right) ; m_{l}$ is the $l$ th component of $\bar{m}$.

Note here that (2.7) implies

$$
\mathbb{E}\left[\sup _{t \leq T} \sup _{m \in B} \sup _{\mu \in \mathscr{P}(B)}\left(1+C\left(m, \mu_{t}\right)\right)^{2}\right] \leq C_{1}<+\infty .
$$

Then we have the martingale

$$
\widetilde{M}_{t}^{n}=\left\langle f, \mu_{t}^{n}\right\rangle-\left\langle f, \mu_{0}^{n}\right\rangle-\int_{0}^{t} A\left(X_{s}\right)\left(\frac{1}{n} \sum_{i=1}^{n} h^{l}\right) d s .
$$


6 Stock market dynamics created by interacting agents

The increasing process of this martingale is given by

$$
\left\langle\widetilde{M}^{n}, \widetilde{M}^{n}\right\rangle_{t}=\int_{0}^{t} \int_{B} \frac{1}{n^{2}} \sum_{i=1}^{n}\left[f(m)-f\left(\mu_{i}(s)\right)\right]^{2} C\left(m, \mu_{s}^{n}, X_{s}\right) \rho(d m) d s .
$$

Define, for a bounded and continuous function $G(\cdot)$ on $B$, the functional $\varphi(G)$ by

$$
\varphi(G)(u)=\log \int_{B} \exp \{m u+G(m)\} \rho(d m), \quad u \in \mathbb{R} .
$$

We get

$$
\partial_{u} \varphi(G)(u) \exp \{\varphi(G)(u)\}=\int_{B} m \exp \{m u+G(m)\} \rho(d m) .
$$

This gives us the following result.

Theorem 3.1. Let $\mu_{0}^{n}$ converge in law to a probability measure $\mu_{0}=h_{0} d \rho \in \mathscr{P}(B)$, where $h_{0} \in L^{\infty}(B)$. Fix the trajectory $\left(X_{t}\right)_{t \in[0, T]}$. Then the process $\left(\mu_{t}^{n}\right)_{t \geq 0}$ converges in law to $h_{t} d \rho$, where the density $h_{t} \in L^{\infty}(B)$ is solution of the functional differential equation

$$
\frac{d h_{t}(m)}{d t}=\exp \left\{\alpha+g_{t}(m)+m \cdot X_{t}\right\}-h_{t}(m) \exp \left\{\alpha+\varphi\left(g_{t}\right)\left(X_{t}\right)\right\}
$$

with initial value $h_{0}$.

Here

$$
g_{t}(\cdot)=\int_{B} g(\cdot, \tilde{m}) h_{t}(\tilde{m}) \rho(d \tilde{m})
$$

Proof. (1) Since $h_{0} d \rho$ is the limit of $\mu_{0}^{n} \in \mathscr{P}(B)$, we have $h_{0} \geq 0$ and $\int h_{0} d \rho=1$. We can easily see that $h_{t}(m) \geq 0$ and

$$
\begin{aligned}
\sup _{t} \operatorname{ess} \sup _{m} h_{t}(m) & \leq \underset{m}{\operatorname{ess} \sup _{0}} h_{0}(m)+\int_{0}^{T} \exp \left\{\alpha+g_{s}(m)+m \cdot X_{s}\right\} d s \\
& \leq\left\|h_{0}\right\|_{\infty}+T \cdot \text { const } \cdot \sup _{s \leq T} \exp \left\{\widetilde{\operatorname{const}} X_{s}\right\},
\end{aligned}
$$

because $g_{t}(m)$ is bounded by $\|g\|_{\infty}$. Thus, by (2.7)

$$
\mathbb{E}\left[\sup _{t}\left\|h_{t}\right\|_{\infty}\right]<+\infty
$$

Equations (3.12), (3.13) show also the uniform differentiability of $h_{t}(m)$ with respect to $t \in[0, T]$. Moreover, $\int h_{t} d \rho=1$ implies $(d / d t) \int h_{t} d \rho=0$, thus $\int h_{t} d \rho=1$ for all $t \in$ $[0, T]$. 
(2) As the set $\mathscr{P}(B)$ is compact for the weak-star topology, to show tightness of the process $\left(\mu_{t}^{n}\right)_{t \geq 0}$, it is enough to show the uniform continuity in the following form: for all $f \in \mathscr{C}(B)$ and all $\eta>0, \epsilon>0$, there exist $n_{0} \in \mathbb{N}$ and $\delta>0$ such that

$$
\sup _{n \geq n_{0}} \sup _{0 \leq \tau_{1} \leq \tau_{2} \leq\left(\tau_{1} \wedge \delta\right)} \mathbb{P}^{n}\left\{\left|\left\langle f, \mu_{\tau_{2}}^{n}\right\rangle-\left\langle f, \mu_{\tau_{1}}^{n}\right\rangle\right|>\eta\right\} \leq \epsilon
$$

where $\langle\cdot, \cdot\rangle$ are the duality brackets and $\tau_{1}, \tau_{2}$ are stopping times.

From (3.6), we have

$$
\left\langle f, \mu_{\tau_{2}}^{n}\right\rangle-\left\langle f, \mu_{\tau_{1}}^{n}\right\rangle=\widetilde{M}_{\tau_{2}, \tau_{1}}^{n}+\int_{\tau_{1}}^{\tau_{2}} \int_{B}\left[\left\langle f, \delta_{m}\right\rangle-\left\langle f, \mu_{s}^{n}\right\rangle\right] C\left(m, \mu_{s}^{n}, X_{s}\right) \rho(d m) d s
$$

where $\widetilde{M}_{\tau_{2}, \tau_{1}}^{n}=\widetilde{M}_{\tau_{2}}^{n}-\widetilde{M}_{\tau_{1}}^{n}$. The second term of the second member is bounded in absolute value by $2 \delta\|f\|_{\infty} C_{1}$ for $n$ sufficiently big. By Tchebychev inequality, we get

$$
\begin{aligned}
\mathbb{P}^{n}\left\{\left|\left\langle f, \mu_{\tau_{2}}^{n}\right\rangle-\left\langle f, \mu_{\tau_{1}}^{n}\right\rangle\right|>\eta\right\} \\
\leq \eta^{-2} \mathbb{E}^{n}\left[\left(\left\langle f, \mu_{\tau_{2}}^{n}\right\rangle-\left\langle f, \mu_{\tau_{1}}^{n}\right\rangle\right)^{2}\right] \\
\leq 2 \eta^{-2} \mathbb{E}^{n}\left[\left(\frac{1}{n} \sum_{i=1}^{n} \int_{\tau_{1}}^{\tau_{2}} \int_{B}\left[\left|f(m)-f\left(\mu_{i}(s)\right)\right|\right] C\left(m, \mu_{s}^{n}, X_{s}\right) \rho(d m) d s\right)^{2}\right] \\
\quad+2 \eta^{-2} \mathbb{E}^{n}\left[\frac{1}{n^{2}} \sum_{i=1}^{n} \int_{\tau_{1}}^{\tau_{2}} \int_{B}\left[f(m)-f\left(\mu_{i}(s)\right)\right]^{2} C\left(m, \mu_{s}^{n}, X_{s}\right) \rho(d m) d s\right] \\
\leq 8 \eta^{-2} \delta^{2}\|f\|_{\infty}^{2} C_{1}^{2}+8 \eta^{-2} n^{-1} \delta\|f\|_{\infty}^{2} C_{1},
\end{aligned}
$$

which is less than $\epsilon$ for all $n \in \mathbb{N}$ and $\delta$ sufficiently small. This shows (3.14).

(3) We will characterize the limit process of $\left(\mu_{t}^{n}\right)_{t \geq 0}$.

We have by Kolmogorov inequality

$$
\begin{aligned}
\mathbb{P}^{n}\left\{\sup _{t \leq T}\left|\int_{0}^{t} \int_{B^{n}}\left[\frac{1}{n} \sum_{l=1}^{n}\left(h^{l}(\bar{m})-h^{l}\left(\mu_{s^{-}}^{(n)}\right)\right)\right] \tilde{\Lambda}(d \bar{m}, d s)\right|>\epsilon\right\} \\
\leq \epsilon^{-2} \sup _{t \leq T} \mathbb{E}^{n}\left[\left(\int_{0}^{t} \int_{B^{n}}\left[\frac{1}{n} \sum_{l=1}^{n}\left(h^{l}(\bar{m})-h^{l}\left(\mu_{s^{-}}^{(n)}\right)\right)\right] \tilde{\Lambda}(d \bar{m}, d s)\right)^{2}\right] \\
\leq 4 \epsilon^{-2} n^{-1}\|f\|_{\infty}^{2} C_{1} T .
\end{aligned}
$$

Hence

$$
\mathbb{P}^{n}\left\{\sup _{t \leq T}\left|\int_{0}^{t} \int_{B^{n}}\left[\frac{1}{n} \sum_{l=1}^{n}\left(h^{l}(\bar{m})-h^{l}\left(\mu_{s^{-}}^{(n)}\right)\right)\right] \tilde{\Lambda}(d \bar{m}, d s)\right|>\epsilon\right\}=\mathscr{O}\left(n^{-1}\right) .
$$


8 Stock market dynamics created by interacting agents

Then outside a set of uniformly small probability, we have

$$
\begin{aligned}
\left\langle f, \mu_{t}^{n}\right\rangle= & \left\langle f, \mu_{0}^{n}\right\rangle+\int_{0}^{t}\left\langle f, \exp \left\{\alpha+\int_{B} g(\cdot, \tilde{m}) \mu_{s}^{n}(d \tilde{m})+\cdot X_{s}\right\} d \rho\right\rangle d s \\
& -\int_{0}^{t}\left\langle f, \mu_{s}^{n}\right\rangle \exp \left\{\alpha+\varphi\left(\int_{B} g(\cdot, \tilde{m}) \mu_{s}^{n}(d \tilde{m})\right)\left(X_{s}\right)\right\} d s+O\left(n^{-1}\right) .
\end{aligned}
$$

The last term of (3.19) converges uniformly in probability to zero. Thus any limit process $\left(\mu_{t}\right)_{t \geq 0}$ of $\left(\mu_{t}^{n}\right)_{t \geq 0}$ satisfies

$$
\left\langle f, \mu_{t}\right\rangle=\left\langle f, \mu_{0}\right\rangle+\int_{0}^{t}\left\langle f, C\left(\cdot, X_{s}\right) d \rho\right\rangle d s-\int_{0}^{t}\left\langle f, \mu_{s}\right\rangle \exp \left\{\alpha+\varphi\left(g_{s}(\cdot)\right)\left(X_{s}\right)\right\} d s,
$$

where

$$
\begin{aligned}
C\left(m, \mu_{t}, X_{t}\right) & =\exp \left\{\alpha+g_{t}(m)+m \cdot X_{t}\right\}, \\
g_{t}(m) & =\int_{B} g(m, \tilde{m}) \mu_{t}(d \tilde{m}) .
\end{aligned}
$$

We will show now that (3.20) has a unique solution on $[0, T]$. We fix a trajectory $\left(X_{t}\right)_{0 \leq t \leq T}$, which is almost surely bounded. Set for $\mu \in \mathscr{P}(B)$,

$$
H_{t}(\mu)=C(\cdot, \mu) d \rho-\mu\langle C(\cdot, \mu), d \rho\rangle
$$

Let us show that $H_{t}(\mu)$ is a Lipschitz function with respect to $\mu$. Let $\mu$ and $\nu$ be two measures satisfying (3.20), then we have for any function $f \in \mathscr{C}(B)$,

$$
\begin{aligned}
\left|\left\langle f, H_{t}(\mu)-H_{t}(\nu)\right\rangle\right| & |\langle f, C(\cdot, \mu) d \rho-\mu\langle C(\cdot, \mu), d \rho\rangle\rangle-(\langle f, C(\cdot, \nu) d \rho-\nu\langle C(\cdot, v), d \rho\rangle\rangle)| \\
= & \mid\left\langle f, e^{\alpha+m \cdot X_{t}}\left(e^{\langle g(m, \cdot), \mu\rangle}-e^{\langle g(m, \cdot), \nu\rangle}\right) \rho(d m)\right\rangle \\
& -\langle f, \mu-\nu\rangle\left\langle e^{\alpha+m \cdot X_{t}} e^{\langle g(m, \cdot), \mu\rangle}, \rho(d m)\right\rangle \\
& -\langle f, \nu\rangle\left\langle e^{\alpha+m \cdot X_{t}}\left(e^{\langle g(m, \cdot), \mu\rangle}-e^{\langle g(m, \cdot), \nu\rangle}\right), \rho(d m)\right\rangle \mid \\
\leq & \left|\left\langle(f-\langle f, \nu\rangle) e^{\alpha+m \cdot X_{t}}\left(e^{\langle g(m, \cdot), \mu\rangle}-e^{\langle g(m, \cdot), \nu\rangle}\right), \rho(d m)\right\rangle\right| \\
& +\left|\left\langle e^{\alpha+m \cdot X_{t}} e^{\langle g(m, \cdot), \mu\rangle}, \rho(d m)\right\rangle\right||\langle f, \mu-\nu\rangle| \\
\leq & 2\|f\|_{\infty} \cdot C_{1} \cdot \int_{B}|\langle g(m, \cdot), \mu-\nu\rangle| \rho(d m)+2\|g\|_{\infty} \cdot C_{2} \cdot|\langle f, \mu-\nu\rangle| .
\end{aligned}
$$

Set $f(\cdot)=g(m, \cdot)$, then

$$
\int_{B}\left|\left\langle g(m, \cdot), H_{t}(\mu)-H_{t}(\nu)\right\rangle\right| \rho(d m) \leq C_{3} \int_{B}|\langle g(m, \cdot), \mu-\nu\rangle| \rho(d m) .
$$


Define the seminorm on $\mathscr{P}(B)$ by $\|\mu\|=\sup _{m \in B}|\langle g(m, \cdot), \mu\rangle|$. Thus

$$
\begin{aligned}
& \left|\left\langle f, H_{t}(\mu)-H_{t}(\nu)\right\rangle\right| \\
& \quad \leq 2\|f\|_{\infty} \cdot C_{1} \cdot \int_{B}|\langle g(m, \cdot), \mu-\nu\rangle| \rho(d m)+2\|g\|_{\infty} \cdot C_{2} \cdot|\langle f, \mu-\nu\rangle| .
\end{aligned}
$$

Replacing $f(\cdot)$ by $g(m, \cdot)$ and changing the $\sup _{m \in B}$, we get

$$
\left\|H_{t}(\mu)-H_{t}(\nu)\right\| \leq C_{4}\|\mu-\nu\|
$$

where $C_{4}$ is a constant depending on $g$.

This shows, by Gronwall's lemma that for two solutions $\mu_{t}=h_{t}^{1} d \rho$ and $v_{t}=h_{t}^{2} d \rho$ of (3.10) we have

$$
\left\langle g(m, \cdot), \mu_{t}\right\rangle=\left\langle g(m, \cdot), v_{t}\right\rangle
$$

it follows from (3.20) that for any function $f \in \mathscr{C}(B)$,

$$
\left|\left\langle f, H_{t}(\mu)-H_{t}(\nu)\right\rangle\right| \leq C\left|\left\langle f, \mu_{t}-v_{t}\right\rangle\right|
$$

Second application of Gronwall's lemma will show the uniqueness of the solution.

3.2. Convergence of price process. In this section, we rewrite the definition (2.5) in the following way:

$$
S_{t}^{n}=S_{0} \cdot \mathscr{E}_{t} X \cdot \cdot\left\langle\exp \{\cdot t\}, \mu_{t}^{n}\right\rangle
$$

Since the integral is a bounded continuous function, we get immediately the following result.

Corollary 3.2. Under the assumptions of Theorem 3.1, the process $\left(S_{t}^{n}\right)_{t \geq 0}$ converges in law, when $n$ goes to infinity, to the process $\left(S_{t}\right)_{t \geq 0}$ defined by

$$
S_{t}=S_{0} \cdot \mathscr{E}_{t} X \cdot \cdot\left\langle\exp \{\cdot t\}, \mu_{t}\right\rangle .
$$

Proof. The proof follows directly from Theorem 3.1.

Remark 3.3. Of course, with $\rho(d m)=\delta_{\mu}(d m)$, hence $h_{t}(m)=1_{\{\mu\}}(m)$, and $X_{t}=\sigma B_{t}$, where $B_{t}$ is a standard Brownian motion, we get the price process considered in BlackScholes model. 


\section{Evolution of stock price in a nervous market}

We know that when the market is very nervous, which is defined in our model by the reaction intensity $\alpha$ going to infinity, stock prices may become discontinuous under certain conditions. In this case we speak about a stock market crash. Historically, crashes appear mostly when stock prices are dropping. This is in accordance with our model, since the condition of high nervousness ( $\alpha$ going to infinity) is only realistic when the prices are already decreasing for some time. To study the convergence of the price process $\left(S_{t}\right)_{t \geq 0}$ when $\alpha$ goes to infinity, we must first study the convergence of the interaction process $\left(g_{t}(m)\right)_{t \geq 0}$, where $g_{t}(m)=\int_{B} g(m, \tilde{m}) h_{t}(\tilde{m}) \rho(d \tilde{m})$, in this case.

Theorem 4.1. When $\alpha$ goes to infinity, the process $\left(g_{t}(m)\right)_{t \geq 0}$ converges to the process $\left(\hat{g}_{t}(m)\right)_{t \geq 0}$ such that

$$
\hat{g}_{t}(m)=\int_{B} g(m, \tilde{m}) \hat{h}_{t}(\tilde{m}) \rho(\tilde{m})
$$

where $\hat{h}_{t}(\tilde{m})$ satisfies the steady state equation

$$
\hat{h}_{t}(\tilde{m})=\frac{\exp \left\{\hat{g}_{t}(m)+\tilde{m} \cdot X_{t}\right\}}{\exp \left\{\varphi\left(\hat{g}_{t}(\cdot)\right)\left(X_{t}\right)\right\}}
$$

Remark 4.2. Keeping $X_{t} \equiv x$ fixed, there may be situations where (4.1) and (4.2) may not have unique solution. In general, however, these are isolated points almost surely. An additional right continuity condition will give us unique solutions.

From (4.2) we can deduce an autonomous equation for $\hat{g}_{t}(m)$, which is of interest with respect to the characterization of the price process $\hat{S}_{t}$ in the limit $\alpha \rightarrow \infty$,

$$
\hat{g}_{t}(m)=\frac{\int_{B} g(m, \tilde{m}) \exp \left\{g_{t}(\tilde{m})+\tilde{m} \cdot X_{t}\right\} \rho(d \tilde{m})}{\exp \left\{\varphi\left(\hat{g}_{t}\right)\left(X_{t}\right)\right\}},
$$

$\varphi$ being the functional defined by (3.8) and (3.9). Then

$$
\widehat{S}_{t}=S_{0} \cdot \mathscr{E}_{t} X \cdot \exp \left\{\varphi\left(\hat{g}_{t}(\cdot)\right)\left(X_{t}+t\right)-\varphi\left(\hat{g}_{t}(\cdot)\right)\left(X_{t}\right)\right\}
$$

Example 4.3. We are going now to study (4.3), and for that we will consider a particular case by taking $B=[b, h]$, where $b, h \in \mathbb{R}$ with $b<h$ and the distribution $\rho=(1 / 2)\left(\delta_{b}+\right.$ $\left.\delta_{h}\right)$. Moreover, let $g(m, \tilde{m})=\beta \cdot m \cdot \tilde{m}$. Let $\nu=(b+h) / 2$ and $\delta=(h-b) / 2$.

With $u=\beta \hat{m}_{t}+X_{t}$, we get the well-known steady state (4.3) in the form

$$
\hat{m}_{t}=\left\{\begin{array}{l}
\frac{1}{\beta}\left(u-X_{t}\right) \\
\nu+\delta \tanh (\delta u) .
\end{array}\right.
$$

The system (4.5) has exactly one solution if $\delta^{2} \beta \leq 1$, that is, if $\beta \leq 1 / \delta^{2}$. However, if $\beta>1 / \delta^{2}$, (4.5) has one or three solutions, depending on the value of $\beta v+X_{t}$. Thus the market price is not uniquely determined. This indeterminateness of prices is a characteristic phenomenon in incomplete markets. 
Example 4.4. Let $S_{t}^{(n)}$ be the price process in the market defined by $\log S_{t}^{(n)}=(1 /$ n) $\sum_{i=1}^{n} \log S_{t}\left(\mu_{i}\right)$. A similar definition was proposed by Föllmer and Schweizer [2]. Let us reconsider the previous studies for this case, and conserve the external perturbation $X_{t}$ a semimartingale null in zero. The process $\left(S_{t}^{n}\right)_{t \geq 0}$ converges in law to the process $\left(S_{t}\right)_{t \geq 0}$ defined by

$$
S_{t}=S_{0} \cdot \mathscr{E}_{t} X \cdot \exp \left\{m_{t} \cdot t\right\}
$$

where as before $m_{t}=\int_{B} m h_{t}(m) \rho(d m)$ is the limit process of the empirical mean $m_{t}^{n}=$ $(1 / n) \sum_{i=1}^{n} \mu_{i}(t)$, with $h_{t}$ from (3.10).

Remark 4.5. The last result can be compared with those given by Föllmer and Schweizer (see [2, (2.18) and Theorem 3.1]). In both models, the equilibrium price process is a logarithmic mean of individual price assumptions. The differences between the two models are twofold. In their model, the global stochastic perturbations result from individual uncertainties, caused by liquidity demands. For simplicity, this quantity is not incorporated in our model. Instead, we start with a common external perturbation process, explained by conjuncture variations, political events, and so on. Second, we give rather explicit description of the influence of these external events to the individual price assumptions, and of the reciprocal influence of the agents among themselves.

\section{References}

[1] F. Black and M. Scholes, The pricing of options and corporate liabilities, Journal of Political Economy 81 (1973), 637-659.

[2] H. Föllmer and M. Schweizer, A microeconomic approach to diffusion models for stock prices, Mathematical Finance 3 (1993), no. 1, 1-23, "Erratum" Mathematical Finance 4, 285.

[3] J. M. Harrison and S. R. Pliska, Martingales and Stochastic Integrals in the Theory of Continuous Trading, Evanston, Northwestern University, 1981.

[4] P. A. Samuelson, Rational theory of Warrant pricing, Industrial Management Review 6 (1965), $13-31$.

Mohamed Riad Remita: Département de Mathématiques, Faculté des Sciences,

Université Badji Mokhtar, BP 12, 23000 Annaba, Algeria

E-mail address: r_remita@yahoo.fr

Karl-Theodor Eisele: Pôle Européen de Gestion et d'Economie (PEGE), 61 avenue de la Forêt Noire, 67085 Strasbourg Cedex; Faculté des Sciences Economiques et de Gestion,

Université Louis Pasteur, 67070 Strasbourg Cedex, France

E-mail address: eisele@math.u-strasbg.fr 


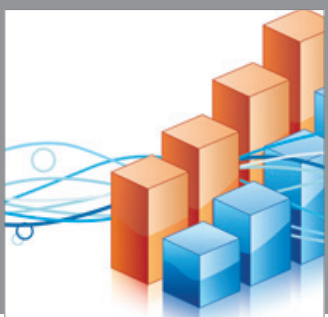

Advances in

Operations Research

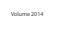

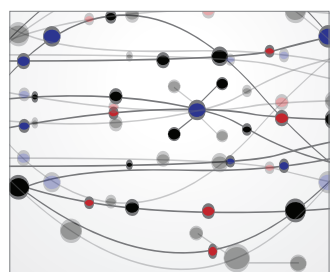

\section{The Scientific} World Journal
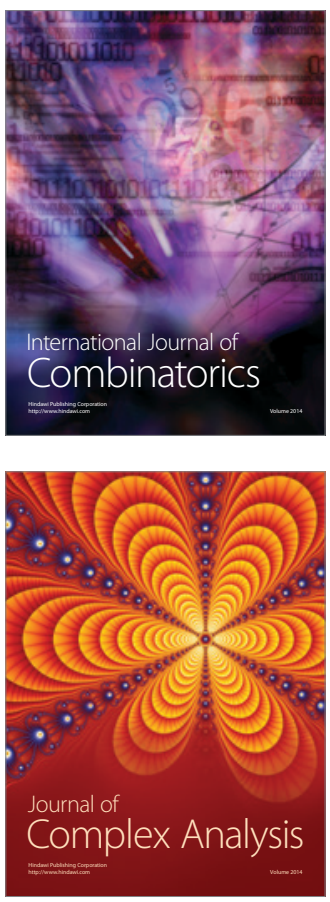

International Journal of

Mathematics and

Mathematical

Sciences
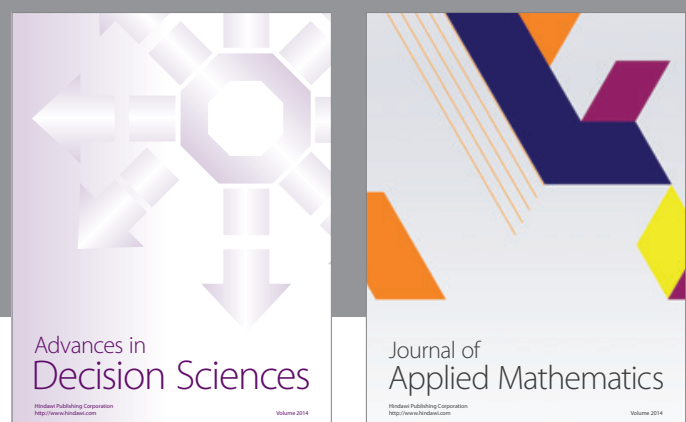

Journal of

Applied Mathematics
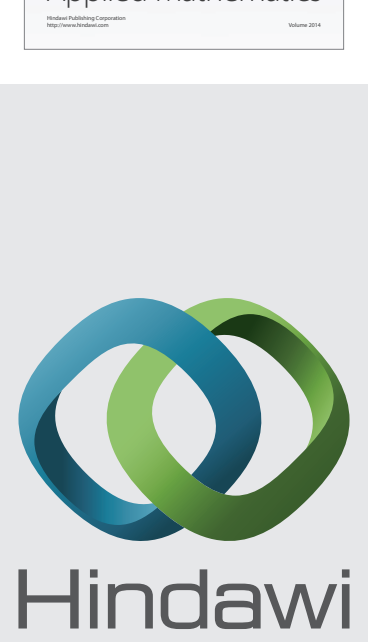

Submit your manuscripts at http://www.hindawi.com
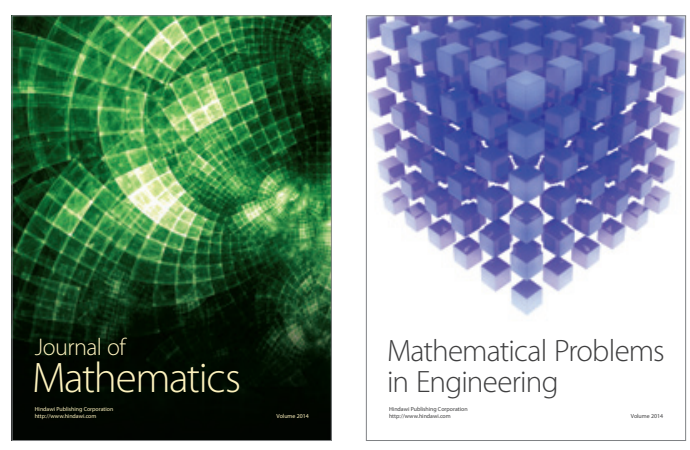

Mathematical Problems in Engineering
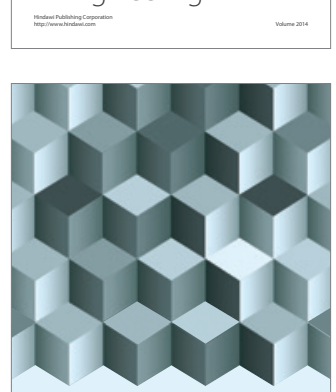

Journal of

Function Spaces
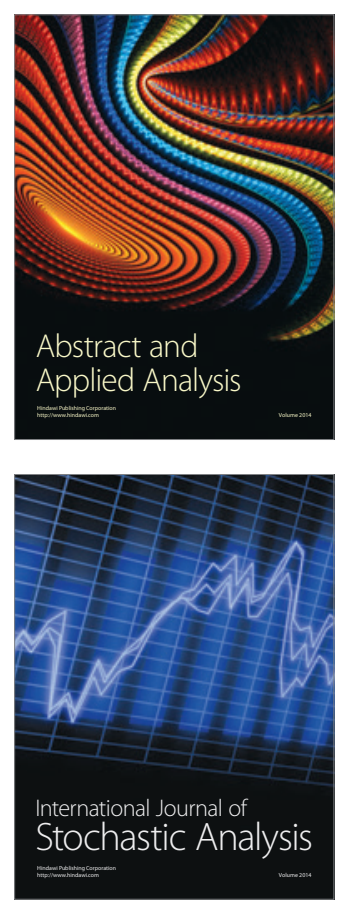

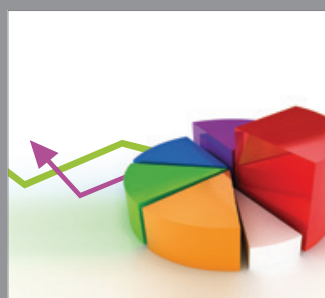

ournal of

Probability and Statistics

Promensencen
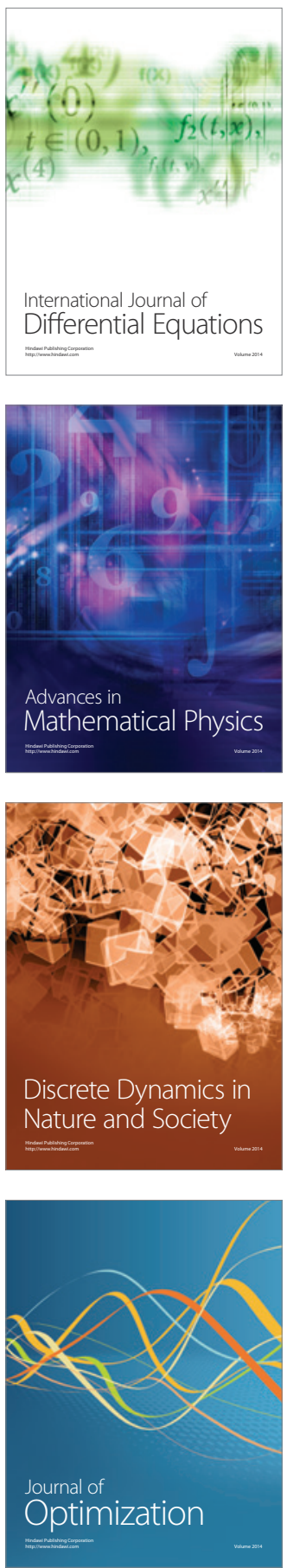